\title{
Muertes por lesiones de tránsito en Argentina: un análisis espacial para el período 2001-2009
}

\author{
Carlos M. Leveau ${ }^{1}$ y Clotilde Ubeda ${ }^{2}$
}

Forma de citar Leveau CM, Ubeda C. Muertes por lesiones de tránsito en Argentina: un análisis espacial para el período 2001-2009. Rev Panam Salud Publica. 2012;31(5):439-42.

RESUMEN Las lesiones de tránsito representan la causa más frecuente de muerte por causas externas en Argentina y un problema de creciente magnitud para la salud pública a nivel global. Los principales objetivos de este trabajo son establecer el nivel de autocorrelación espacial experimentado a nivel de departamentos e identificar la conformación de agrupamientos mediante el cálculo de los indicadores locales de asociación espacial a nivel nacional. Los resultados mostraron un nivel de autocorrelación significativamente positivo en Argentina. Al relacionar las tasas de mortalidad por lesiones de tránsito con la densidad poblacional, se registró un nivel de autocorrelación espacial negativo. Se observó también que la mortalidad por lesiones de tránsito podría representar un problema más grave fuera de las grandes aglomeraciones.

Palabras clave Accidentes de tránsito; heridas y traumatismos; causas externas; sistemas de información geográfica; Argentina.

Las muertes por lesiones de tránsito constituyen un problema de salud pública a nivel global. Según datos de la Organización Mundial de la Salud (OMS), cada año mueren por esta causa más de 1,2 millones de personas, de las cuales $91 \%$ corresponde a países de ingresos bajos y medios (1). En Argentina, datos de 2007 indican que las muertes por causas externas en el país ocupan el primer lugar en el grupo etario de 1 a 44 años, y dentro de este grupo, las debidas a lesiones de tránsito representan $44 \%$ - la causa más frecuente de muerte por lesiones (2, 3). Más todavía, la tasa de mortalidad por lesiones de tránsito du-

\footnotetext{
1 Universidad Nacional de Mar del Plata, Argentina. La correspondencia se debe dirigir a Carlos M. Leveau, cmleveau@mdp.edu.ar

2 INE, "Dr. J.H. Jara” ANLIS Malbran, Mar de Plata, Argentina.
}

rante el período 2001-2009 subió de 9,96 a 10,86 muertes por 100000 habitantes. Estas cifras representan un problema de creciente magnitud para la salud pública en Argentina.

Persona y tiempo han sido las dos dimensiones histórica y preponderantemente utilizadas en la investigación epidemiológica, con poca atención prestada al lugar o al espacio (4). A nivel mundial, durante la última década son pocos los estudios que han analizado diferencias regionales en las muertes por lesiones de tránsito a escala nacional (5-9). Más aún, estos trabajos sobre variaciones geográficas han sido llevados a cabo sobre todo en los países desarrollados.

Los estudios mencionados intentaron explorar y establecer relaciones entre ciertas variables socio-ambientales y las muertes por lesiones por tránsito. En algunos casos analizaron, con resultados dispares, el rol de la densidad poblacional y del nivel de urbanización en la incidencia de muertes por estas lesiones $(6,9)$. Otra manera de estudiar las diferencias geográficas en las muertes por lesiones de tránsito es a través de la autocorrelación espacial, que mide el patrón de distribución de los valores de una variable en un área asociados a valores de la misma u otra variable en áreas vecinas. Si los valores de un conjunto de áreas (agrupamiento) tienden a ser similares, el nivel de autocorrelación espacial será positivo, caso contrario será negativo (10, 11). En epidemiología la autocorrelación espacial es útil para la detección de agrupamientos (clusters) (4).

Hasta el momento, en Argentina no se han realizado análisis espaciales cuantitativos de las muertes por lesiones de tránsito, y no se conoce la autocorrelación espacial ni la relación entre morta- 
lidad por lesiones de tránsito y la densidad poblacional. El presente trabajo se propone precisamente 1) establecer el nivel de autocorrelación espacial experimentado en departamentos de Argentina para el período 2001-2009; 2) identificar la conformación de agrupamientos -en este caso altas tasas de mortalidad por lesiones de tránsito localizadas en unidades espaciales contiguas - mediante el cálculo de los indicadores locales de asociación espacial; 3) examinar el grado de autocorrelación espacial bivariada entre la mortalidad por lesiones de tránsito y la densidad poblacional a nivel nacional y 4) detectar la conformación de agrupamientos locales tomando ambas variables.

Se utilizaron dos fuentes de datos. Una fue la Dirección de Estadísticas e Información en Salud, que tiene cobertura nacional completa y codifica con la Clasificación Internacional de Enfermedades, 10a Revisión (CIE-10) (12). La otra, con datos sobre densidad poblacional, fue el Instituto Nacional de Estadística y Censos (13). Los datos sobre mortalidad por lesiones de tránsito fueron analizados de acuerdo a los códigos de la CIE-10 para el período estudiado. Las unidades geográficas estuvieron conformadas por el mínimo territorio administrativo a nivel nacional (denominado "partido" en la provincia de Buenos Aires y "departamento" en el resto de las provincias) donde se pueden obtener datos sobre defunciones. La tasa de mortalidad por lesiones de tránsito se estimó por 100000 habitantes/año, ${ }^{3}$ agrupándose de acuerdo al lugar de ocurrencia del evento. Para el cálculo de tasas se excluyeron los casos en que la víctima residía fuera de la provincia de ocurrencia del accidente.

En la Ciudad Autónoma de Buenos Aires, se asignó una tasa global debido a que no hay datos desagregados disponibles para los 21 distritos escolares que la componen. El nivel de autocorrelación espacial se calculó con base en el índice I de Moran y fue aplicado tanto a nivel de partidos como de departamentos. El rango de valores posibles que arroja este índice es de -1 a 1 , donde valores positivos indican un agrupamiento espacial de valores similares (máxima concentración) y valores negativos indican un agrupamiento de valores disí-

\footnotetext{
A nivel departamental no se contó con otros denominadores, como el número de vehículos matriculados o los kilómetros recorridos por habitante.
}

miles (máxima dispersión). La relación entre las unidades geográficas vecinas se midió mediante la contigüidad tipo Queen, que considera a todos los vecinos de cada unidad espacial. Para poner a prueba la hipótesis de no independencia entre las observaciones y autocorrelación espacial, se realizaron permutaciones que dieron como resultado distribuciones al azar. Si el valor del índice $I$ de Moran se encontraba en las colas de este conjunto de distribuciones, se rechazaba la hipótesis nula de independencia entre las observaciones. El nivel de significancia del test fue inferior a 0,05 , empleando 499 permutaciones.

Dado que el índice $I$ de Moran - por ser una medida de autocorrelación global- no muestra la localización de los agrupamientos, se calcularon versiones locales denominadas indicadores locales de asociación espacial (LISA, por sus siglas en inglés). Estos indicadores presentan cuatro situaciones: i) agrupamientos de departamentos con valores de tasas por muertes de tránsito 'alto-alto'; ii) agrupamientos de departamentos con valores 'alto-bajo' (área de alto valor de mortalidad por lesiones de tránsito rodeada por áreas con valores bajos de la misma variable o de densidad poblacional); iii) agrupamientos de departamentos con valores 'bajo-alto' (área de bajo valor de mortalidad por lesiones de tránsito rodeada por áreas con valores altos de la misma variable o de densidad poblacional), y iv) agrupamientos con departamentos con tasas 'bajo-bajo'. Celemín (11) aporta una descripción detallada y minuciosa del procedimiento de autocorrelación espacial y los indicadores locales de asociación espacial. Se utilizó el paquete de cómputo Geoda, desarrollado por el Centro de Análisis Geoespacial y Computación, de la Universidad Estatal de Arizona (Estados Unidos).

Al observar el mapa con la distribución nacional de las muertes por lesiones de tránsito, se distinguieron diferentes áreas con altas tasas de mortalidad: una franja oriental en la provincia de Buenos Aires, pero que excluye al "aglomerado" Buenos Aires, las zonas centrales de Chubut y Santa Cruz, el sudoeste de Neuquén, el norte de San Luis y el este de Mendoza y parte de Jujuy (figura 1).

Se registró una autocorrelación espacial significativamente positiva, indicando una tendencia al agrupamiento de tasas de mortalidad por lesiones de tránsito similares (índice $I$ de Moran $=0,29$,
FIGURA 1. Distribución espacial de las muertes por lesiones de tránsito (tasa por 100000 habitantes), Argentina, 2001-2009

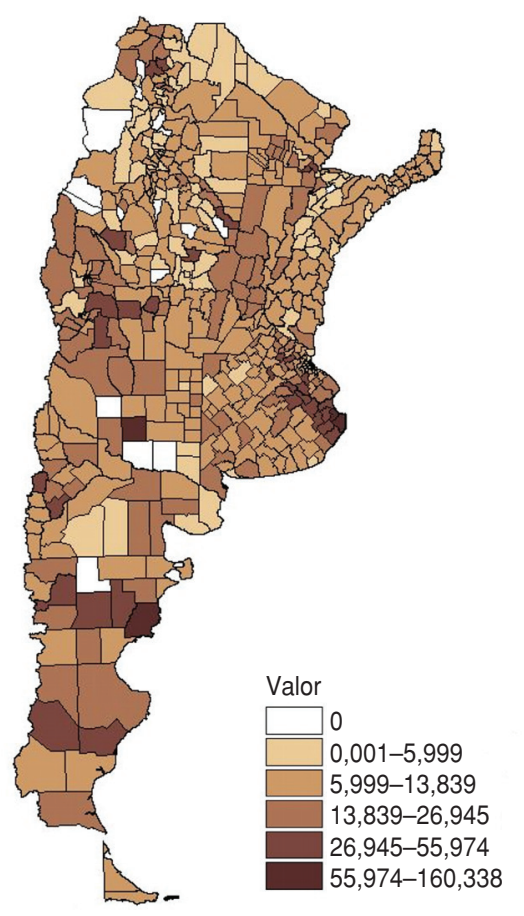

Fuente: elaboración de los autores.

$P<0,05,499$ permutaciones). Los indicadores locales de asociación espacial localizaron los diferentes tipos de agrupamientos, distinguiéndose cuatro grandes agrupamientos de áreas con altas tasas de mortalidad por lesiones de tránsito. En el centro del país estos agrupamientos se localizaron en Mendoza-San Luis y el este de Buenos Aires, mientras que en el sur argentino se localizaron en el centro de Chubut y norte de Santa Cruz (figura 2). Por otro lado, se observaron cuatro grandes áreas de baja mortalidad por estas lesiones, de las cuales tres se ubicaron en el norte del país: en Corrientes, Salta-Formosa y un área conformada por departamentos de Catamarca, Salta y Tucumán. La cuarta área se localizó en el Gran Buenos Aires, conformado por sus partidos, incluida la Ciudad Autónoma de Buenos Aires, y cuya superficie ocupa total o parcialmente la mancha urbana de dicho aglomerado (figura 2).

El test de autocorrelación espacial bivariado entre la tasa de mortalidad por lesiones de tránsito y la densidad poblacional exhibió una autocorrelación significativamente negativa entre las dos variables (índice $I$ de Moran $=-0,15$, $P<0,05,499$ permutaciones). En general 
FIGURA 2. Indicadores locales de asociación espacial de la variable muertes por lesiones de tránsito, Argentina, 2001-2009

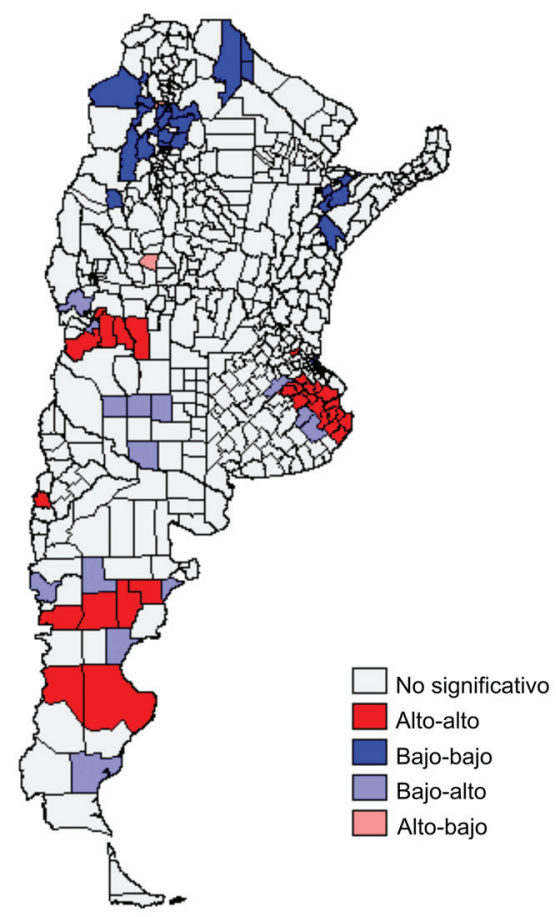

Fuente: elaboración de los autores.

se encontró que los departamentos con altas tasas de mortalidad por lesiones de tránsito estaban rodeados por departamentos con baja densidad poblacional (figura 3).

En el caso de la provincia de Buenos Aires, aunque los partidos que conforman el área de alta mortalidad por lesiones de tránsito se caracterizan por tener bajas densidades poblacionales, transitan por sus territorios rutas que conectan al Gran Buenos Aires con los principales centros turísticos del país. En el resto del país los departamentos que conformaron los agrupamientos de alta mortalidad tampoco registraron altas densidades poblacionales. Son departamentos que no pertenecen a las grandes aglomeraciones del interior del país, sino que en la mayoría de los casos constituyen departamentos con rutas que conectan a las principales ciudades del subsistema de asentamientos de cada provincia. De manera exploratoria, el patrón espacial detectado permite hipotetizar factores que pueden estar más relacionados a variables ambientales, como estado de las rutas, condiciones climáticas, escaso auxilio y centros de salud alejados. Los resultados de este estudio también parecen
FIGURA 3. Indicadores locales de asociación espacial bivariados entre las muertes por lesiones de tránsito (2001-2009) y la densidad poblacional (2001), Argentina

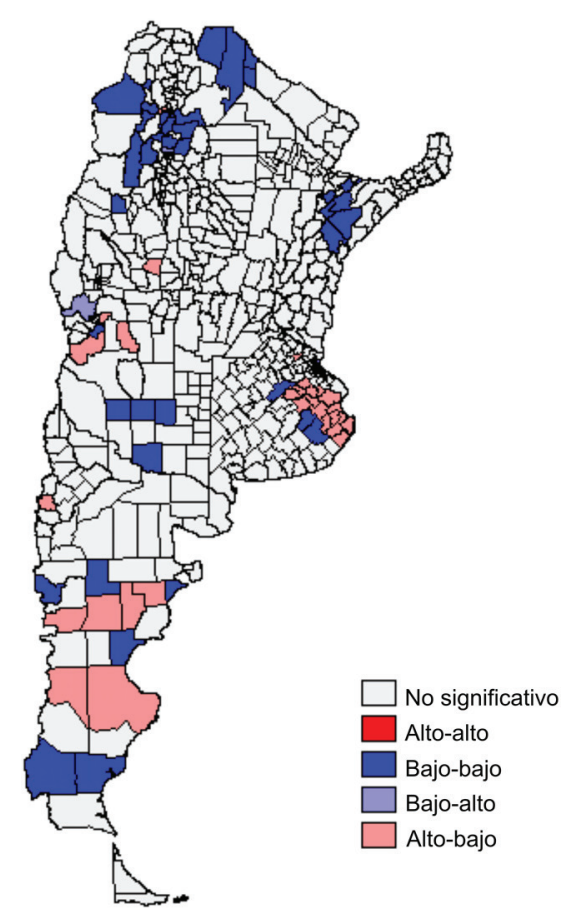

Fuente: elaboración de los autores.

indicar que la mortalidad por lesiones de tránsito representaría un problema más grave fuera de las grandes aglomeraciones. En tal sentido, Lambert y Meyer (14) encontraron que en las áreas metropolitanas del sudeste de Estados Unidos las tasas de mortalidad por lesiones de tránsito se relacionaron negativamente con la densidad poblacional, es decir, fueron mayores en las áreas periurbanas con respecto a las áreas urbanas más densamente pobladas. En el presente estudio, abarcando un área de análisis mayor, también se registró una relación negativa entre ambas variables.

El patrón de autocorrelación espacial positivo observado coincide con el notificado por Erdogan (9) en Turquía, donde las provincias fueron consideradas como unidades geográficas. Los agrupamientos de alta mortalidad por lesiones de tránsito se localizaron en provincias turísticas o que sirven como corredores de transporte entre las provincias más pobladas. Este fenómeno guarda cierta similitud con el caso argentino, ya que, como se mencionó, uno de los mayores agrupamientos de alta mortalidad se localiza en los partidos que unen el Gran
Buenos Aires, la zona más poblada de la Argentina, con los destinos turísticos balnearios del sudeste de la Provincia de Buenos Aires.

El presente estudio planteó algunas limitaciones. En primer lugar, no se pudieron analizar los patrones de distribución de las muertes por lesiones de tránsito al interior de la Ciudad Autónoma de Buenos Aires, la zona más densamente poblada del país. La incorporación de estos datos, no disponibles, podría haber cambiado la configuración del agrupamiento conformado por el Gran Buenos Aires. En segundo lugar, la imposibilidad de considerar los kilómetros recorridos por habitante, o el número de vehículos matriculados, como denominadores de la mortalidad por accidentes de tránsito impide establecer otros patrones de distribución geográfica para las nuevas tasas resultantes. Asimismo, debería haber un sesgo de selección teniendo en cuenta que al incluir en el análisis a las personas de una misma provincia, algunas zonas turísticas como la de Los Lagos (provincia de Neuquén), que presentan una baja densidad poblacional, pudieron presentar altas tasas de lesionados debido a que se incluyeron personas que no residen en el departamento respectivo, pero pertenecen a la misma provincia.

Los resultados obtenidos mostraron un nivel de autocorrelación significativamente positivo en Argentina. Al relacionar las tasas de mortalidad por accidentes de tránsito con la densidad poblacional, se registró un nivel de autocorrelación espacial negativo.

El análisis geográfico de la mortalidad provocada por tránsito con sistemas de información geográfica permitió identificar las áreas de mayor mortalidad a nivel departamental. La identificación de estas áreas puede dar lugar a estudios observacionales complementarios que ayuden a comprender mejor las circunstancias en que se producen las lesiones y constituye un insumo fundamental para el desarrollo de programas preventivos (15).

Agradecimientos. Los autores quieren dar las gracias al Ministerio de Salud de la Nación, por brindar sus bases de datos de mortalidad para el presente estudio. También expresan su gratitud a Andrea Perinetti, Jorge Ungaro y cuatro revisores anónimos cuyas contribuciones mejoraron sustancialmente la calidad de este trabajo. 


\section{REFERENCIAS}

1. Organización Mundial de la Salud. Informe sobre la situación mundial de la seguridad vial: es hora de pasar a la acción. Ginebra, Suiza: OMS; 2009

2. Ubeda C, Bhalla K, Puthenpurakal J, EspitiaHardeman V, Dellinger A, Ferrante D, et al. Lesiones por tránsito en Argentina. Disponible en: http://roadinjuries.globalburdenof injuries.org/argentina Acceso el 4 de marzo de 2012.

3. Ubeda C, Espitia-Hardeman V, Bhalla K, Borse NN, Abraham JP, Dellinger A, et al. National burden of road traffic injuries in Argentina. Int J Inj Contr Saf Promot. 2011;6:1-10.

4. Moore DA, Carpenter TE. Spatial analytical methods and geographic information systems: use in health research and epidemiology. Epidemiol Rev. 1999;21(2):143-61.

5. Híjar M, Vázquez-Vela E, Arreola-Risa C. Pedestrian traffic injuries in Mexico: a country update. Inj Control Saf Promot. 2003;10(1-2): 37-43.

6. La Torre G, van Beeck E, Quaranta G, Mannocci A, Ricciardi W. Determinants of within-country variation in traffic accident mortality in Italy: a geographical analysis. Int J Health Geogr. 2007;6:49.

7. Rivas-Ruiz F, Perea-Milla E, Jiménez-Puente A. Geographic variability of fatal road traffic injuries in Spain during the period 2002-2004: an ecological study. BMC Public Health. 2007; 7:266.

8. Jones AP, Haynes R, Kennedy V, Harvey IM, Jewell T, Lea D. Geographical variations in mortality and morbidity from road traffic accidents in England and Wales. Health Place. 2008;14(3):519-35.

9. Erdogan S. Explorative spatial analysis of traffic accident statistics and road mortality among the provinces of Turkey. J Safety Res. 2009;40(5):341-51.

10. Waller LA, Gotway CA. Applied spatial statistics for public health data. New Jersey, U.S.A.: John Wiley \& Sons; 2004.

11. Celemín JP. Autocorrelación espacial e indicadores locales de asociación espacial. Importancia, estructura y aplicación. Rev Univ Geogr. 2009;18(1):11-31.
12. Ministerio de Salud de la Nación, Argentina. Sistemas de Información de salud. 2004. Disponible en: http://www.deis.gov.ar/ Acceso el 4 de marzo de 2012.

13. República Argentina, Ministerio de Economía y Producción, Secretaría de Política Económica, Instituto Nacional de Estadística y Censos (INDEC). Proyecciones provinciales de población por sexo y grupos de edad 2001-2015. Serie $\mathrm{N}^{\circ} 31$ Análisis demográfico. Disponible en: http://www.indec.mecon.ar/ Acceso el 4 de marzo de 2012.

14. Lambert TE, Meyer PB. Ex-urban sprawl as a factor in traffic fatalities and EMS response times in the southeastern United States. Journal of Economic Issues. 2006;XL(4):941-53.

15. Híjar-Medina MC. Utilidad del análisis en el estudio geográfico de las muertes por atropellamiento. Salud Publica Mex. 2000;42(3): $188-93$.

Manuscrito recibido el 4 de agosto de 2011. Aceptado para publicación, tras revisión, el 23 de febrero de 2012.
ABSTRACT

\section{Deaths from road injuries in Argentina: a spatial analysis for the 2001-2009 period}

Key words
Traffic injuries in Argentina are the most frequent cause of death from external injuries and a public health problem of increasing magnitude at the global level. The objectives of this study are to establish the level of spatial autocorrelation at the department level and identify the formation of groupings by calculating local indicators of spatial association at the national level. The results reveal a significantly positive level of autocorrelation in Argentina. A negative level of spatial autocorrelation was recorded when mortality from road injuries was related to population density. It was also noted that mortality from road injuries could pose a more serious problem outside large urban areas.

Accidents, traffic; wounds and injuries; external causes; geographic information systems; Argentina. 